\title{
Correction to: One-step templated synthesis of chiral organometallic salicyloxazoline complexes
}

Mei Luo ${ }^{1,3^{*}} \mathbb{0}$, Jing Cheng Zhang ${ }^{1}$, Hao Yin'2 ${ }^{2}$ Cheng Ming Wang ${ }^{2}$, Susan Morris-Natschke ${ }^{3}$ and Kuo-Hsiung Lee Le, $^{3 *}$

\section{Correction to: BMC Chem (2019) 13:51}

https://doi.org/10.1186/s13065-019-0565-z

Following publication of the original article [1], the authors reported an error in Schemes 1 and 2 and repeated line in subsection "Bis(ligand) nickel(II) chelate $\left(\mathrm{NiL}_{2}\right)$ ".

Please see below for the revised Schemes 1 and 2 and the corrected paragraph.

\section{Bis(ligand) nickel (II) chelate ( $\mathrm{NiL1}_{2}$ )}

Prepared using the procedure described for compound 1 by refluxing a mixture of 2-cyanophenol (2.3001 g, $19.33 \mathrm{mmol}), \mathrm{Ni}(\mathrm{OAc})_{2} \cdot 4 \mathrm{H}_{2} \mathrm{O}(2.4528 \mathrm{~g}, 9.86 \mathrm{mmol})$ or
$\mathrm{NiCl}_{2} \cdot 6 \mathrm{H}_{2} \mathrm{O}(2.4374 \mathrm{~g}, 10.25 \mathrm{mmol})$ and D-phenylglycinol $(4.2318 \mathrm{~g})$ in $40 \mathrm{~mL}$ of dry chlorobenzene for $60 \mathrm{~h}$. The product was obtained as dark brown crystals $(2.5112 \mathrm{~g}$ in $92 \%$ yield or $2.6949 \mathrm{~g}$ ) in $95 \%$ yield after column chromatography (petroleum ether $/ \mathrm{CH}_{2} \mathrm{Cl}_{2}, 4 / 1$ ). m.p.: $196-198{ }^{\circ} \mathrm{C},[\alpha]_{\mathrm{D}}^{25}=+119.57^{\circ}\left(\mathrm{c}=0.0488, \mathrm{CH}_{3} \mathrm{OH}\right),{ }^{1} \mathrm{H}$ NMR $\left(600 \mathrm{MHz}, \mathrm{CDCl}_{3}\right.$ and DMSO, $\left.27^{\circ} \mathrm{C}\right): 7.85-7.86$ $(\mathrm{m}, 2 \mathrm{H}), 7.22-7.49(\mathrm{~m}, \mathrm{l} 2 \mathrm{H}), 6.46(\mathrm{~d}, \mathrm{~J}=7.3 \mathrm{~Hz}, 2 \mathrm{H}), 6.30$ $(\mathrm{t}, \mathrm{J}=6.4 \mathrm{~Hz}, 2 \mathrm{H}), 5.70-5.98(\mathrm{~m}, 2 \mathrm{H}), 4.54-4.62(\mathrm{~m}, 2 \mathrm{H})$, $4.32-4.41(\mathrm{~m}, 2 \mathrm{H}) ; \delta_{\mathrm{C}}\left(150 \mathrm{MHz}, \mathrm{CDCl}_{3}\right): 164.5,164.4$, $142.3,133.5,127.3,126.0,125.7,124.3,113.1,107.8$, 107.7 $(\times 2), 72.6,72.5,67.0,65.1,65.0 . v_{\max }\left(\mathrm{cm}^{-1}\right): 3453$, 3024, 2906, 1617, 1541, 1475, 1447, 1394, 1349, 1265,
2<smiles>N#Cc1ccccc1O</smiles><smiles>[R]C(N)CO</smiles>
(excess)
$+\mathrm{MCl}_{2} / \mathrm{M}(\mathrm{OAc})_{2} \mathrm{PhCl}$, reflux

(1.0 equiv.)

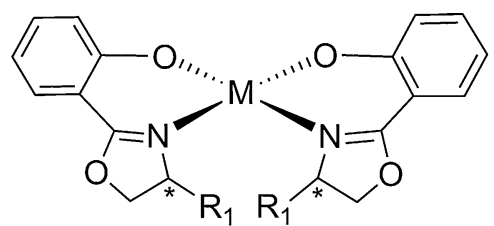

1,2,3: $\mathrm{R}_{1}: \mathrm{D}-\mathrm{Ph}$

1: $\mathrm{Cu}(\mathrm{OAC})_{2}, 65 \%$ or $\mathrm{CuCl}_{2}, 85 \%$

2: $\mathrm{Ni}(\mathrm{OAc})_{2}, 92 \%$ or $\mathrm{NiCl}_{2}, 95 \%$

3: $\mathrm{CoCl}_{2}, 72 \%$

4: $\mathrm{R}_{1}: \mathrm{L}-\mathrm{CH}_{2} \mathrm{Ph} ; \mathrm{PdCl}_{2}, 86 \%$

Scheme 1 Templated synthesis of complexes 1-4

*Correspondence: luomei@pku.edu.cn; khlee@unc.edu

${ }^{3}$ Natural Products Research Laboratories, UNC Eshelman School

of Pharmacy, University of North Carolina, Chapel Hill, NC 27599-7568,

USA

Full list of author information is available at the end of the article 


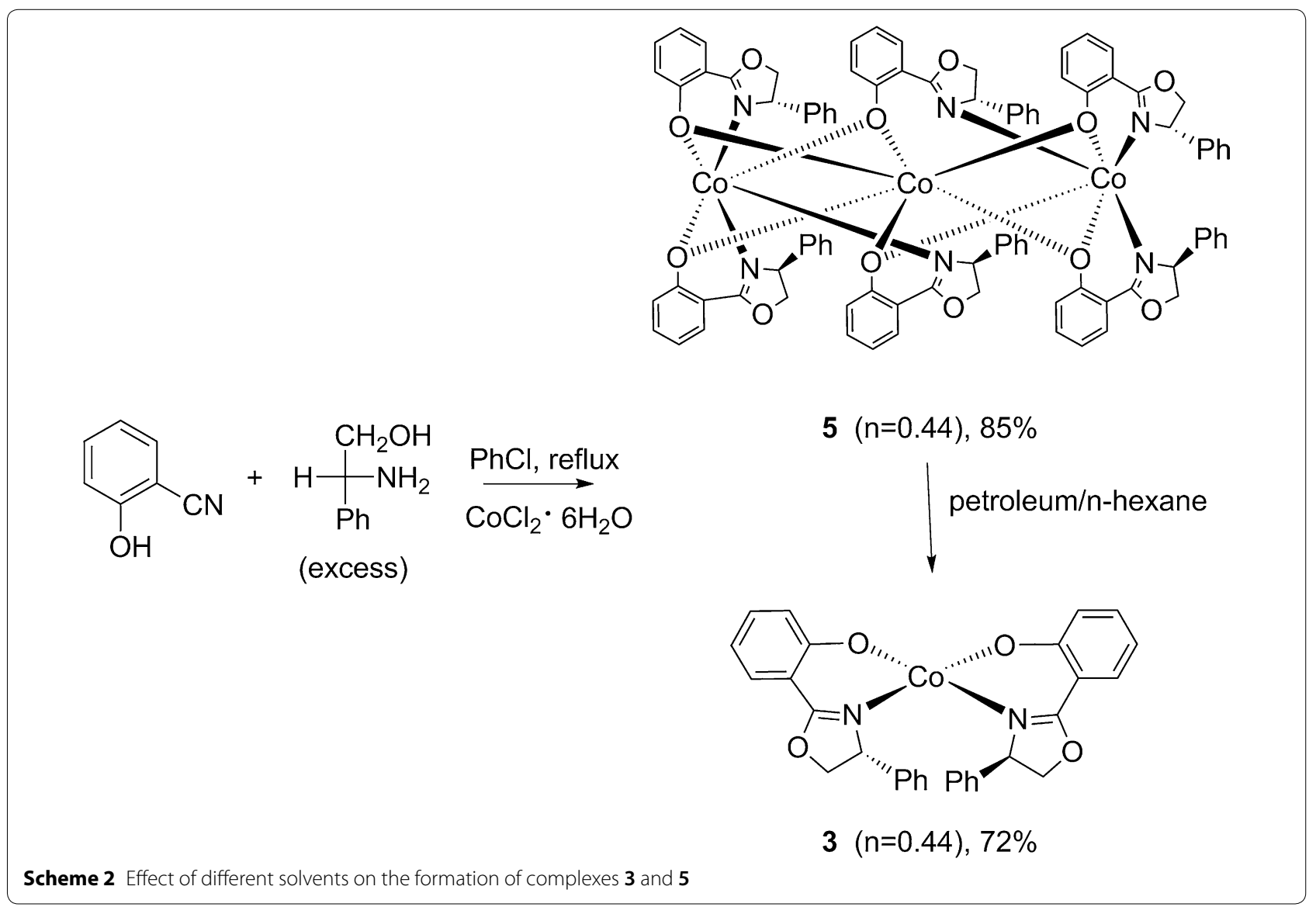

1231, 1154, 1077, 1029, 949, 931, 85,5, 755, 695, 574, 533, 415. Elemental analysis for $\mathrm{C}_{30} \mathrm{H}_{24} \mathrm{~N}_{2} \mathrm{O}_{4} \mathrm{Ni}$ requires $\mathrm{C}$ : 67.32\%, H: 4.52\%, N: 5.23\%; found: C: $67.22 \%$, H: $4.39 \%$, $\mathrm{N}: 5.26 \%$.

\section{Tri(ligand) cobalt chelate $\left(\mathrm{CoL} 1_{3}\right)$}

Prepared using the procedure described for compound $\mathbf{1}$ by refluxing a mixture of $1.5671 \mathrm{~g}$ of $\mathrm{Co}(\mathrm{OAc})_{2} \cdot 4 \mathrm{H}_{2} \mathrm{O}$ (6.29 mmol), 2-cyanophenol (1.7699 g, $14.86 \mathrm{mmol})$ and D-phenylglycinol (3.6798 g) in $40 \mathrm{~mL}$ of dry chlorobenzene for $60 \mathrm{~h}$. The product was obtained in $70 \%$ yield $(2.5424 \mathrm{~g})$ as dark brown crystals after column chromatography (petroleum ether $/ \mathrm{CH}_{2} \mathrm{Cl}_{2}, 4 / 1$ ). m.p.: 174$176{ }^{\circ} \mathrm{C},[\alpha]^{5}{ }_{\mathrm{D}}=-1014.1^{\circ}\left(0.0212, \mathrm{CH}_{3} \mathrm{OH}\right), \delta \mathrm{H}(600$ $\left.\mathrm{MHz}, \mathrm{CDCl}_{3}, 2{ }^{\circ} \mathrm{C}\right) 7.50-7.52(\mathrm{~m}, 1 \mathrm{H}), 7.23-7.24(\mathrm{~m}$, $1 \mathrm{H}), 7.02-7.07(\mathrm{~m}, 2 \mathrm{H}), 6.87-6.97(\mathrm{~m}, 9 \mathrm{H}), 6.74-6.80(\mathrm{~m}$, $7 \mathrm{H}), 6.56(\mathrm{~d}, \mathrm{~J}=8.56 \mathrm{~Hz}, 1 \mathrm{H}), 6.45-6.49(\mathrm{~m}, 3 \mathrm{H}), 6.41(\mathrm{~d}$, $\mathrm{J}=8.5 \mathrm{~Hz}, 1 \mathrm{H}), 6.24-6.27(\mathrm{~m}, 2 \mathrm{H}), 5.45-5.48(\mathrm{~m}, 1 \mathrm{H})$, 5.29-5.32 (m, 1H), 4.91-4.92 (m, 2H), 4.79-4.82 (m, 2H), 4.33-4.36 (m, 1H), 4.26-4.28 (m, 2H); $\delta \mathrm{C}(150 \mathrm{MHz}$, $\left.\mathrm{CDCl}_{3}\right)$ 170.1, 170.0, 168.9, 166.2, 165.3, 164.8, $140.3,139.8,133.1(\times 2), 132.3,128.1,128.0,127.7,127.5$, $127.4,127.1,126.8,125.3,124.4,123.7,122.9,113.9$, 113.5, 113.1), 112.9, 109.2, 107.6, 76.3, 75.8, 75.2, 66.8, 66.1, 63.8. $v_{\max }\left(\mathrm{cm}^{-1}\right): 3448,3061,1617,1583,1541,1468$, 1455, 1442, 1396, 1347, 1265,1225, 1152, 1078, 949, 931, 856, 756, 747, 728, 696, 593, 577, 545, 409. Elemental analysis for $\mathrm{C}_{46} \mathrm{H}_{38} \mathrm{Cl}_{2} \mathrm{~N}_{3} \mathrm{O}_{6} \mathrm{Co}$ requires $\mathrm{C}$ : $64.34 \%, \mathrm{H}$ : 4.46, N: 4.89\%; found: C: $64.48 \%, \mathrm{H}: 4.27, \mathrm{~N}: 4.90 \%$.

\section{Author details}

${ }^{1}$ College of Chemistry and Chemical Engineering, Hefei University of Technology, Hefei 230009, China. ${ }^{2}$ Hefei National Laboratory for Physical Sciences at the Microscale, University of Science and Technology of China, Hefei 230026, China. ${ }^{3}$ Natural Products Research Laboratories, UNC Eshelman School of Pharmacy, University of North Carolina, Chapel Hill, NC 27599-7568, USA. ${ }^{4}$ Chinese Medicine Research and Development Center, China Medical University and Hospital, Taichung 40447, Taiwan.

The original article can be found online at https://doi.org/10.1186/s1306 $5-019-0565-z$ 


\section{Publisher's Note}

Springer Nature remains neutral with regard to jurisdictional claims in published maps and institutional affiliations.

Received: 17 April 2019 Accepted: 17 April 2019

Published online: 10 May 2019

\section{Reference}

1. Luo M, Zhang JC, Yin H, Wang CM, Morris-Natschke S, Lee K-H (2019) One-step templated synthesis of chiral organometallic salicyloxazoline complexes. BMC Chem. 13:51. https://doi.org/10.1186/s13065-019-0565-z

Ready to submit your research? Choose BMC and benefit from:

- fast, convenient online submission

- thorough peer review by experienced researchers in your field

- rapid publication on acceptance

- support for research data, including large and complex data types

- gold Open Access which fosters wider collaboration and increased citations

- maximum visibility for your research: over $100 \mathrm{M}$ website views per year

At $\mathrm{BMC}$, research is always in progress.

Learn more biomedcentral.com/submissions 